\title{
Spindle checkpoint silencing: ensuring rapid and concerted anaphase onset
}

\author{
Kevin G Hardwick ${ }^{1 *}$ and Jagesh V Shah ${ }^{2}$
}

\author{
Addresses: ${ }^{1}$ Wellcome Trust Centre for Cell Biology, University of Edinburgh, King's Buildings, Mayfield Road, Edinburgh, EH9 3JR, UK; \\ ${ }^{2}$ Department of Systems Biology, Harvard Medical School, 4 Blackfan Circle, HIM 564, Boston, MA 02115, USA \\ * Corresponding author: Kevin G Hardwick (kevin.hardwick@ed.ac.uk) \\ Fl000 Biology Reports 2010, 2:55 (doi:10.34I0/B2-55)
}

The electronic version of this article is the complete one and can be found at: http://fl000.com/reports/biology/content/2/55

\begin{abstract}
The spindle checkpoint delays anaphase onset in the presence of defective kinetochore-microtubule attachments. Such delays can last for just a few minutes or several hours, but very shortly after all chromosomes achieve bi-orientation, a remarkably synchronous anaphase ensues. We are beginning to understand the pathways involved in silencing spindle checkpoint signals and subsequent activation of the anaphase-promoting complex. Here, we review recent advances made in our understanding of the molecular mechanisms regulating this critical cell cycle transition.
\end{abstract}

\section{Introduction and context}

Mis-segregation of chromosomes in the germline leads to aneuploidy and spontaneous abortion or birth defects and, in the soma, is associated with several diseases, including cancer [1]. Despite the complexities of mitosis, such segregation defects are in fact extremely rare. The high fidelity of this process is due, in large part, to the action of checkpoints (surveillance systems) that coordinate the successful completion of cell biological processes (DNA replication and spindle assembly) with cell cycle progression. During mitosis, the spindle checkpoint monitors kinetochore-microtubule interactions and delays anaphase onset until all sister-chromatid pairs are attached and bi-oriented on the mitotic spindle [2]. Its components, such as the Mad (mitotic arrest defective) and Bub (budding uninhibited by benomyl) proteins, do this by inhibiting Cdc20, which is an activator of the mitotic E3 ubiquitin ligase known as the anaphase-promoting complex or cyclosome (APC/C). By inhibiting the APC/ $\mathrm{C}$, securin and cyclin $\mathrm{B}$ are stabilized, thereby maintaining sister-chromatid cohesion and high levels of mitotic CDK (cyclin-dependent kinase) activity $[3,4]$.

Checkpoint signals (e.g., Mad2-Cdc20 and mitotic checkpoint complexes, or MCCs) are generated at unattached kinetochores and then must be amplified throughout the cytoplasm to ensure global inhibition of the APC/C (Figure 1). Kinetochores often bind laterally to microtubules at first, but then these microtubule interactions will mature to form the more appropriate stable, end-on, bipolar attachments that 'satisfy' the spindle checkpoint. These attachments are under tension and it is thought that this spatially separates and constrains kinase and phosphatase activities within distinct centromeric and kinetochore regions: such subtleties may be key to determining the overall balance of checkpoint signal production versus silencing.

Once such attachments are achieved, several kinetochore-based factors act coordinately to stop kinetochoremediated Mad2-Cdc20 complex generation (see below and Figure 2). However, preventing Mad2-Cdc20 production at kinetochores is not sufficient and the existing inhibitors in the cytoplasm and any cytoplasmic amplification mechanisms must be rapidly quenched to ensure a timely and synchronous anaphase $[4,5]$.

Do kinetochore and cytoplasmic silencing mechanisms act cooperatively or in distinct pathways? Here, it is important to note that some model systems may be 
Figure I. APC/C inhibition is regulated by signalling within the cytoplasm (a) and at the kinetochore (b)

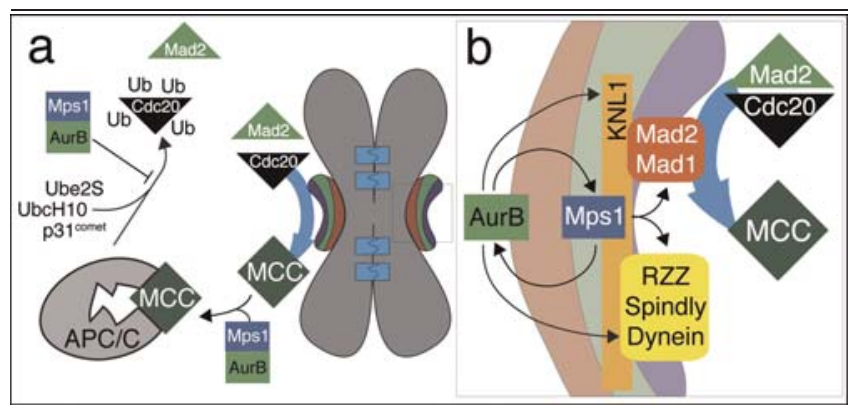

(a) The inhibition of the anaphase-promoting complex/cyclosome (APC/C) occurs primarily in the cytoplasm through the generation and amplification of kinetochore-generated signals that are balanced by the dissociation of inhibitory complexes. Checkpoint kinases (e.g., MpsI and AurB) are thought to act on this dissociation pathway to stabilize the inhibited form of the APC/C. (b) These same kinases act at the kinetochore to activate and maintain the checkpoint through the regulation of the local generation of the wait signal. This activity keeps checkpoint generators such as the MadI/ Mad2 and the Spindly/RZZ/dynein complexes localized to kinetochores through the action of kinases on scaffolds such as kinetochore-null I (KNLI) and checkpoint activators such as dynein. AurB, Aurora B kinase; Mad, mitotic arrest defective; MCC, mitotic checkpoint complex; Mps I, monopolar spindle I; RZZ, rough deal, zeste white 10, zwilch.

Figure 2. Silencing of the checkpoint acts both in the cytoplasm (a) and locally at each kinetochore (b) upon microtubule attachment

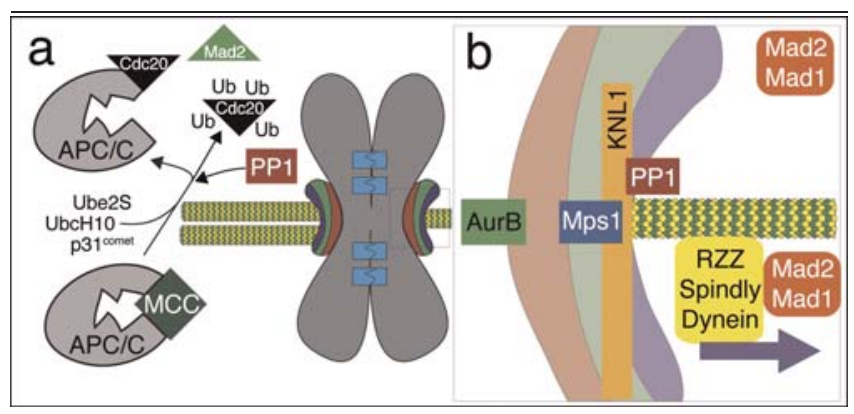

At a single kinetochore, the binding of the microtubule and subsequent recruitment and activation of protein phosphatase I (PPI) locally reduce checkpoint kinase activity and release checkpoint complexes via dissociation of MadI/Mad2 or translocation along microtubules via dynein. Loss of the single generators culminates in the total loss of generation when all chromosomes become attached. The remaining cytoplasmic inhibitory complexes (MCC-APC/C) are dissociated in part by the natural decay in the absence of generation and also by the activation of pathways that enhance dissociation to produce the synchronous onset of anaphase. The dissociation activity results in the activation of the APC/C by $\mathrm{Cdc} 20$, the ubiquitination and degradation of cyclin $B$ and securin, and the onset of anaphase. APC/C, anaphase-promoting complex/cyclosome; AurB, Aurora $B$ kinase; KNLI, kinetochore-null I; Mad, mitotic arrest defective; MCC, mitotic checkpoint complex; Mps I, monopolar spindle I; RZZ, rough deal, zeste white 10, zwilch. more reliant on cytoplasmic (or nucleoplasmic in the closed mitosis of yeast) versus kinetochore-based generation of inhibitory signals. How does silencing differ in these varied systems? In this report, we outline some of the recent advances in our mechanistic understanding of spindle checkpoint silencing.

\section{Major recent advances}

Major recent advances have been made in areas of kinase (Aurora and monopolar spindle 1 [Mps1]) and phosphatase (protein phosphatase 1 [PP1]) signaling, dynein-mediated stripping of checkpoint proteins from kinetochores, and proteolytic turnover of checkpoint components. Other silencing pathways (such as $\mathrm{p} 31^{\text {comet }}$ ) exist, but little has been learned of late with regard to their modes of action.

\section{Balancing kinases/phosphatase activity}

Aurora B and protein phosphatase I

In fission yeast, Aurora kinase 1 (Ark1) activity is required to maintain spindle checkpoint arrest, and when its kinase activity is inhibited (using ark1-as, an ATP analog-sensitive allele), kinetochore localized PP1 activity (Dis2 in Schizosaccharomyces pombe) is also required to silence the checkpoint and reactivate the APC/C [5]. Budding yeast also uses a PP1 homolog (Glc7 in Saccharomyces cerevisae) in silencing the spindle checkpoint [6] in concert with a localization factor, Fin 1 [7]. Most recently, it was demonstrated in vertebrates that the gamma isoform of PP1 is recruited via KNL1 (kinetochore-null 1) binding to kinetochores, where it opposes Aurora B activity to stabilize microtubule attachments [8]. This creates a bi-stability, with either Aurora B or PP1 activity dominating in the outer kinetochore, thereby revealing an important switch to a stabilized kinetochore-microtubule attachment that may initiate kinetochore-mediated spindle checkpoint silencing. Ndc80, MCAK (mitotic centromere-associated kinesin), and KNL1 are all Aurora and PP1 substrates that influence microtubule stabilization, but the substrates that are most important for checkpoint silencing remain to be identified, although KNL1, Ndc80, and dynein are candidates [8-11].

\section{Monopolar spindle I kinase (and opposing PPases)}

Mps1 inhibition is a powerful suppressor of the checkpoint in mammalian cells. RNA interference (RNAi) of Mps1 shortens mitosis [12-14] much like the depletion of Mad2 and BubR1 first demonstrated by Meraldi, Draviam, and Sorger [15]. Mps1 inhibition within mitosis, through the use of small-molecule inhibitors, is equally potent [16-18]. This supports models in which Mps1 kinase carries out functions in 'wait' signal generation at the kinetochore, such as promoting localization of Mad1/Mad2, and also in 
'wait' signal stabilization in the cytoplasm via a mechanism that has yet to be described.

Given the complex phenotypes associated with the loss of these checkpoint kinases, we propose that Aurora and Mps1 kinases are tri-functional (Figure 1) and can (a) regulate microtubule attachments, (b) regulate 'wait' signals at the kinetochore, and (c) stabilize 'wait' signals in the cytoplasm. As chromosomes attach, the reduced local activity of these kinases results in kinetochore composition changes that stabilize the microtubule attachment and reduce wait signal generation (Figure 2).

\section{Loss of kinetochore-localized spindle checkpoint components \\ Dynein/Spindly/RZZ}

Spindly and the RZZ (rough deal, zeste white 10, zwilch) complex are required for dynein recruitment to kinetochores. Upon microtubule attachment, several outer kinetochore components, including Mad1-Mad2, are transported to spindle poles in a dynein-dependent fashion $[19,20]$. This motor-mediated removal of the Mad1-Mad2 scaffold has been proposed to eliminate wait signal generation by splitting the essential catalytic platform into an inactive form [21]. It was recently shown that dyneindependent removal of Spindly from microtubule-attached kinetochores is required for checkpoint silencing. Spindly 'motif' mutants that cannot bind dynein prevent dynein recruitment to kinetochores and result in persistent checkpoint signaling with persistent localization of the Mad1/Mad2 to the attached kinetochore [22]. However, in the complete absence of Spindly, after RNAi-mediated depletion, there exists a mechanism to delocalize Mad1/ Mad2 complexes from attached kinetochores, perhaps revealing a conserved silencing pathway present in lower eukaryotes without obvious Spindly/RZZ homologs.

The removal of the wait signal generator from the attached kinetochore occurs through at least two pathways: dissociation and physical translocation along the attaching microtubules themselves. This disruption of signal generation occurs at each chromosome. After the total number of unattached chromosomes is reduced to zero, the loss of the signal generation is followed by the release of inhibition that is already acting on the APC/C.

\section{Relieving inhibition on the anaphase-promoting complex/cyclosome \\ Ubiquitination activity}

During mitosis, unattached kinetochores generate inhibitory complexes (e.g., MCC) that result in the significant accumulation of inhibitory APC/C complexes (MCC-APC/C) [23-25]. Dissociation of these complexes is required to permit APC/C activation. Work by Reddy and colleagues [26] identified a role for ubiquitination in the dissociation of this complex via the direct ubiquitination of Cdc20. Their work established that the E2 enzyme UbcH10, in concert with p $31^{\text {comet }}$, could act to ubiquitinate Cdc20 in the context of the MCC-APC/C complex, providing the first direct mechanism for relief of APC/C inhibition. However, Cdc20 turnover is also critical for checkpoint arrest [27-29], and a full understanding of the complex regulation of Cdc20 remains to be realized.

More recently, another E2 ubiquitin enzyme, Ube2S, has been shown to be critical for rapid anaphase onset after release from extended drug-induced checkpoint activation. Surprisingly, loss of the enzyme during a normal, unperturbed mitosis has, at best, a modest delay on anaphase onset, indicating a specific role during extended checkpoint activation. Perhaps more importantly, the loss of Ube2S in an extended checkpoint arrest reveals a decoupling between kinetochore-mediated silencing and cytoplasmic APC/C activation [30-32].

A key feature of a synchronous anaphase onset is the coupling of MCC-APC/C complex dissociation (i.e., APC/C activation) to kinetochore attachment, which is proposed here to occur via inhibition of the checkpoint kinases Mps1 and Aurora B kinase (AurB) and activation of PP1. This coupling would speed anaphase onset after final kinetochore attachment, a commonly made and enigmatic observation [4].

\section{Future directions}

Whereas mechanistic details of silencing pathways are being clarified on a monthly basis, the overall control and any coordination of these pathways are far from clear. Inter-module coordination could occur via AurBmediated recruitment of PP1 to KNL1. These activities can feed into regulation of Spindly-dynein transport as kinetochore targeting of dynein is under PP1 regulation [11]. However, non-stripping pathways of Mad1-Mad2 release must also be regulated, and identification of other AurB/Mps1/PP1 targets both at kinetochores and in the cytoplasm will be required. Finally, the wealth of quantitative timelapse and biochemical measurements provide a robust substrate for computational modeling that will have a major role in testing the many molecular hypotheses.

\section{Abbreviations}

APC/C, anaphase-promoting complex/cyclosome; Ark1, Aurora kinase 1; AurB, Aurora B kinase; Bub, budding uninhibited by benomyl; KNL1, kinetochore-null 1; Mad, mitotic arrest defective; MCC, mitotic checkpoint complex; Mps1, monopolar spindle 1; PP1, protein 
phosphatase 1; RNAi, RNA interference; RZZ, rough deal, zeste white 10 , zwilch.

\section{Competing interests}

The authors declare that they have no competing interests.

\section{Acknowledgments}

We thank members of the Hardwick and Shah labs for engaging discussions. Research on the spindle assembly checkpoint is supported by grants from the Wellcome Trust (083610) to KGH and the National Institutes of Health (GM077238) to JVS.

\section{References}

I. Holland AJ, Cleveland DW: Boveri revisited: chromosomal instability, aneuploidy and tumorigenesis. Nat Rev Mol Cell Biol 2009, 10:478-87.

2. Burke DJ, Stukenberg PT: Linking kinetochore-microtubule binding to the spindle checkpoint. Dev Cell 2008, I4:474-9.

3. Musacchio A, Salmon ED: The spindle-assembly checkpoint in space and time. Nat Rev Mol Cell Biol 2007, 8:379-93.

4. Ciliberto A, Shah JV: A quantitative systems view of the spindle assembly checkpoint. EMBO J 2009, 28:2 I62-73.

5. Vanoosthuyse $V$, Hardwick KG: A novel protein phosphatase I-dependent spindle checkpoint silencing mechanism. Curr Biol 2009, |9:||76-8|.

\section{FI000 Factor 3.2 Recommended}

Evaluated by Laura Trinkle-Mulcahy 22 Jul 2009, Domenico Grieco 29 Sep 2009

6. Pinsky BA, Nelson CR, Biggins S: Protein phosphatase I regulates exit from the spindle checkpoint in budding yeast. Curr Biol 2009, 19: I 182-7.

\section{FI000 Factor 3.2 Recommended}

Evaluated by Laura Trinkle-Mulcahy 22 Jul 2009, Andy Golden 05 Aug 2009

7. Akiyoshi B, Nelson CR, Ranish JA, Biggins S: Quantitative proteomic analysis of purified yeast kinetochores identifies a PPI regulatory subunit. Genes Dev 2009, 23:2887-99.

FI000 Factor 6.0 Must Read

Evaluated by Kevin Hardwick 02 Dec 2009

8. Liu D, Vleugel M, Backer CB, Hori T, Fukagawa T, Cheeseman IM, Lampson MA: Regulated targeting of protein phosphatase I to the outer kinetochore by KNLI opposes Aurora B kinase. J Cell Biol 2010, I 88:809-20.

FI000 Factor 6.5 Must Read

Evaluated by Kevin Hardwick 18 Mar 2010, Laura Trinkle-Mulcahy 25 Mar 2010, Stefan Westermann 26 Mar 2010

9. Deluca JG, Gall WE, Ciferri C, Cimini D, Musacchio A, Salmon ED: Kinetochore microtubule dynamics and attachment stability are regulated by Hecl. Cell 2006, I 27:969-82.

FI000 Factor 6.9 Must Read

Evaluated by Jonathan Scholey I 5 Dec 2006, Claire Walczak I5 Dec 2006, Kevin Hardwick 19 Dec 2006, Bruce McEwen 03 Jan 2007, Harold Erickson 23 Jan 2007

10. Kemmler S, Stach M, Knapp M, Ortiz J, Pfannstiel J, Ruppert T, Lechner J: Mimicking Ndc80 phosphorylation triggers spindle assembly checkpoint signalling. EMBO J 2009, 28:1099-I 10.

FI000 Factor 3.0 Recommended

Evaluated by Kevin Hardwick 08 Jun 2009
II. Whyte J, Bader JR, Tauhata SB, Raycroft M, Hornick J, Pfister KK, Lane WS, Chan GK, Hinchcliffe EH, Vaughan PS, Vaughan KT: Phosphorylation regulates targeting of cytoplasmic dynein to kinetochores during mitosis. J Cell Biol 2008, I 83:819-34.

12. Stucke VM, Silljé HHW, Arnaud L, Nigg EA: Human Mps I kinase is required for the spindle assembly checkpoint but not for centrosome duplication. EMBO J 2002, 21:1723-32.

13. Jelluma $N$, Brenkman A, van den Broek N, Cruijsen C, van Osch M, Lens S, Medema R, Kops G: Mps I phosphorylates Borealin to control Aurora B activity and chromosome alignment. Cell 2008, I 32:233-46.

FI000 Factor 6.5 Must Read

Evaluated by Kevin Hardwick 04 Feb 2008, Domenico Grieco 07 Feb 2008, William Earnshaw 10 Jul 2008

14. Tighe A, Staples O, Taylor S: Mps I kinase activity restrains anaphase during an unperturbed mitosis and targets Mad2 to kinetochores. J Cell Biol 2008, I81:893-90I.

15. Meraldi P, Draviam VM, Sorger PK: Timing and checkpoints in the regulation of mitotic progression. Dev Cell 2004, 7:45-60.

FI000 Factor 6.5 Must Read

Evaluated by Timothy Yen 20 Jul 2004, Kevin Hardwick 09 Aug 2004, Bruce McEwen 14 Sep 2004

16. Schmidt M, Budirahardja Y, Klompmaker R, Medema RH: Ablation of the spindle assembly checkpoint by a compound targeting MpsI. EMBO Rep 2005, 6:866-72.

17. Kwiatkowski $N$, Jelluma $N$, Filippakopoulos $P$, Soundararajan $M$, Manak MS, Kwon M, Choi HG, Sim T, Deveraux QL, Rottmann S, Pellman D, Shah JV, Kops G], Knapp S, Gray NS: Small-molecule kinase inhibitors provide insight into Mps I cell cycle function. Nat Chem Biol 2010, 6:359-68.

18. Sliedrecht T, Zhang C, Shokat KM, Kops GJPL: Chemical genetic inhibition of Mpsl in stable human cell lines reveals novel aspects of Mps I function in mitosis. PLOS ONE 20I0, 5:el025I.

19. Howell BJ, McEwen BF, Canman JC, Hoffman DB, Farrar EM, Rieder CL, Salmon ED: Cytoplasmic dynein/dynactin drives kinetochore protein transport to the spindle poles and has a role in mitotic spindle checkpoint inactivation. J Cell Biol 200 I, I 55: I I 59-72.

FI000 Factor 3.0 Recommended

Evaluated by Duane Compton 16 Jan 2002

20. Basto R, Scaerou F, Mische S, Wojcik E, Lefebvre C, Gomes R, Hays T, Karess R: In vivo dynamics of the rough deal checkpoint protein during Drosophila mitosis. Curr Biol 2004, I4:56-6I.

FI000 Factor 3.0 Recommended Evaluated by David Sharp 06 Feb 2004

21. Shah JV, Botvinick E, Bonday Z, Furnari F, Berns M, Cleveland DW: Dynamics of centromere and kinetochore proteins; implications for checkpoint signaling and silencing. Curr Biol 2004, 14:942-52.

FI000 Factor 3.0 Recommended Evaluated by Claire Walczak 22 Jun 2004

22. Gassmann R, Holland A, Varma D, Wan X, Civril F, Cleveland DW, Oegema K, Salmon ED, Desai A: Removal of Spindly from microtubule-attached kinetochores controls spindle checkpoint silencing in human cells. Genes Dev 20I0, 24:957-7I.

23. Sudakin V, Chan GK, Yen TJ: Checkpoint inhibition of the APC/C in HeLa cells is mediated by a complex of BUBRI, BUB3, CDC20, and MAD2. J Cell Biol 200I, I 54:925-36.

FI000 Factor 4.8 Must Read

Evaluated by Joan Ruderman 07 Nov 200I, Erich Nigg 29 Oct 200I

24. Hardwick KG, Johnston RC, Smith DL, Murray AW: MAD3 encodes a novel component of the spindle checkpoint which interacts with Bub3p, Cdc20p, and Mad2p. J Cell Biol 2000, I48:87I-82.

25. Sczaniecka M, Feoktistova A, May K, Chen J, Blyth J, Gould K, Hardwick K: The spindle checkpoint functions of Mad3 and 
Mad2 depend on a Mad3 KEN box-mediated interaction with Cdc20-APC/C. J Biol Chem 2008, 283:23039-47.

26. Reddy SK, Rape M, Margansky WA, Kirschner MW: Ubiquitination by the anaphase-promoting complex drives spindle checkpoint inactivation. Nature 2007, 446:92I-5.

FI000 Factor 6.4 Must Read

Evaluated by Robert Fisher 25 Apr 2007, Domenico Grieco 08 May 2007

27. Pan J, Chen RH: Spindle checkpoint regulates Cdc20p stability in Saccharomyces cerevisiae. Genes Dev 2004, I8:|439-5I.

FI000 Factor 6.0 Must Read

Evaluated by Kevin Hardwick 13 Aug 2004

28. King EM, van der Sar SJ, Hardwick KG: Mad3 KEN boxes mediate both Cdc20 and Mad3 turnover, and are critical for the spindle checkpoint. PLoS ONE 2007, 2:e342.
29. Nilsson J, Yekezare M, Minshull J, Pines J: The APC/C maintains the spindle assembly checkpoint by targeting Cdc20 for destruction. Nat Cell Biol 2008, I0:14II-20.

FI000 Factor 6.6 Must Read

Evaluated by Kevin Hardwick 20 Nov 2008, Domenico Grieco 09 Dec 2008, Silke Hauf II Dec 2008

30. Garnett MJ, Mansfeld J, Godwin C, Matsusaka T, Wu J, Russell P, Pines J, Venkitaraman AR: UBE2S elongates ubiquitin chains on APC/C substrates to promote mitotic exit. Nat Cell Biol 2009, II:I363-9.

3I. Williamson A, Wickliffe KE, Mellone BG, Song L, Karpen GH, Rape M: Identification of a physiological E2 module for the human anaphase-promoting complex. Proc Natl Acad Sci U S A 2009, 106:182/3-8.

32. Wu T, Merbl Y, Huo Y, Gallop JL, Tzur A, Kirschner MW: UBE2S drives elongation of KII-linked ubiquitin chains by the anaphasepromoting complex. Proc Nat Acad Sci U S A 20 I0, 107: 1355-60. 